\title{
Financial incentives for smoking cessation in pregnancy: randomised controlled trial
}

\author{
(c) $\frac{(1)(8)}{\mathrm{gy}}$ OPEN ACCESS
}

\begin{abstract}
David Tappin professor ${ }^{1}$, Linda Bauld professor ${ }^{2}$, David Purves research fellow ${ }^{3}$, Kathleen Boyd lecturer ${ }^{4}$, Lesley Sinclair trial manager ${ }^{2}$, Susan MacAskill research fellow ${ }^{2}$, Jennifer McKell research fellow $^{2}$, Brenda Friel health improvement senior ${ }^{5}$, Alex McConnachie senior lecturer ${ }^{6}$, Linda de Caestecker director of public health ${ }^{5}$, Carol Tannahill director ${ }^{7}$, Andrew Radley consultant in public health ${ }^{8}$, Tim Coleman professor ${ }^{9}$, for the Cessation in Pregnancy Incentives Trial (CPIT) Team

'PEACH Unit, Child Health, Glasgow University, Yorkhill, Glasgow G3 8SJ, UK; ${ }^{2}$ Institute for Social Marketing and UK Centre for Tobacco and Alcohol Studies, University of Stirling, Stirling, UK; ${ }^{3}$ Strathclyde University, Glasgow, UK; ${ }^{4}$ Health Economics and Health Technology Assessment Unit, Institute of Health and Wellbeing, Glasgow University, Glasgow, UK; ${ }^{5}$ Public Health, NHS Greater Glasgow and Clyde, Glasgow, UK; ${ }^{6}$ Robertson Centre for Biostatistics and Glasgow Clinical Trials Unit, Glasgow University, Glasgow, UK; ${ }^{7}$ Glasgow Centre for Population Health, Glasgow, UK; ${ }^{8}$ Directorate of Public Health, NHS Tayside, Dundee, UK; ${ }^{9}$ Division of Primary Care and, UK Centre for Tobacco and Alcohol Studies, University of Nottingham, Nottingham, UK
\end{abstract}

\begin{abstract}
Objective To assess the efficacy of a financial incentive added to routine specialist pregnancy stop smoking services versus routine care to help pregnant smokers quit.

Design Phase II therapeutic exploratory single centre, individually randomised controlled parallel group superiority trial.

Setting One large health board area with a materially deprived, inner city population in the west of Scotland, United Kingdom.

Participants 612 self reported pregnant smokers in NHS Greater Glasgow and Clyde who were English speaking, at least 16 years of age, less than 24 weeks pregnant, and had an exhaled carbon monoxide breath test result of $7 \mathrm{ppm}$ or more. 306 women were randomised to incentives and 306 to control.

Interventions The control group received routine care, which was the offer of a face to face appointment to discuss smoking and cessation and, for those who attended and set a quit date, the offer of free nicotine replacement therapy for 10 weeks provided by pharmacy services, and four, weekly support phone calls. The intervention group received routine care plus the offer of up to $£ 400$ of shopping vouchers: $£ 50$ for attending a face to face appointment and setting a quit date; then another $£ 50$ if at four weeks' post-quit date exhaled carbon monoxide confirmed quitting; a further $£ 100$ was provided for continued validated abstinence of exhaled carbon monoxide after 12 weeks; a final £200 voucher was provided for validated abstinence of exhaled carbon monoxide at 34-38 weeks' gestation.
\end{abstract}

Main outcome measure The primary outcome was cotinine verified cessation at 34-38 weeks' gestation through saliva $(<14.2 \mathrm{ng} / \mathrm{mL})$ or urine $(<44.7 \mathrm{ng} / \mathrm{mL})$. Secondary outcomes included birth weight, engagement, and self reported quit at four weeks.

Results Recruitment was extended from 12 to 15 months to achieve the target sample size. Follow-up continued until September 2013. Of the 306 women randomised, three controls opted out soon after enrolment; these women did not want their data to be used, leaving 306 intervention and 303 control group participants in the intention to treat analysis. No harms of financial incentives were documented. Significantly more smokers in the incentives group than control group stopped smoking: 69 (22.5\%) versus $26(8.6 \%)$. The relative risk of not smoking at the end of pregnancy was 2.63 (95\% confidence interval 1.73 to 4.01 ) $\mathrm{P}<0.001$. The absolute risk difference was $14.0 \%$ (95\% confidence interval $8.2 \%$ to $19.7 \%$ ). The number needed to treat (where financial incentives need to be offered to achieve one extra quitter in late pregnancy) was 7.2 (95\% confidence interval 5.1 to 12.2$)$. The mean birth weight was $3140 \mathrm{~g}$ (SD $600 \mathrm{~g}$ ) in the incentives group and 3120 (SD 590) $g$ in the control group ( $P=0.67)$.

Conclusion This phase II randomised controlled trial provides substantial evidence for the efficacy of incentives for smoking cessation in pregnancy; as this was only a single centre trial, incentives should now be tested in different types of pregnancy cessation services and in different parts of the United Kingdom.

Trial registration Current Controlled Trials ISRCTN87508788.

\section{Introduction}

Of 125000 spontaneous miscarriages that occur each year in the United Kingdom, ${ }^{1} 25000^{1}$ are associated with smoking during pregnancy, assuming that $17 \%$ of women smoke during 
pregnancy, ${ }^{2}$ and the relative risk of spontaneous miscarriage compared with non-smokers is $1.2 .{ }^{3}$ If causality were accepted, then with a $20 \%$ increase in risk, 5000 miscarriages each year would be attributable to smoking during pregnancy. Stillbirths occur at a rate of four for every 1000 live births, and 4-7\% are attributable to smoking during pregnancy, ${ }^{4}$ accounting for approximately 180 deaths each year in the United Kingdom. Infant deaths also occur at a rate of 4 per 1000 live births, ${ }^{5}$ and $3-5 \%{ }^{6}$ are attributable to smoking during pregnancy-113 infant deaths each year. In deprived areas, a third of excess stillbirths and postnatal deaths are explained by smoking during pregnancy. ${ }^{7}$ The total annual cost to the UK National Health Service of smoking during pregnancy is estimated to range between $£ 8.1 \mathrm{~m}(\$ 12.3 \mathrm{~m} ; € 10.4 \mathrm{~m})$ and $£ 64 \mathrm{~m}$ for treating the resulting problems in mothers, and between $£ 12 \mathrm{~m}$ and $£ 23.5 \mathrm{~m}$ in infants (aged 0-12 months). ${ }^{8}$

Smoking is a major preventable cause of premature maternal mortality, with lifelong benefits of quitting for children. In Scotland, $70 \%$ of women have at least one baby, ${ }^{9}$ making pregnancy an opportunity to help most women quit before their health is permanently compromised. In $2009,24 \%$ of pregnant women who attended their first antenatal booking appointment in Scotland self reported being smokers, ${ }^{10}$ but only one in 10 used evidence based NHS cessation services, with 3\% reporting abstinence at four weeks after quitting. ${ }^{11}$ In 2010 , the National Institute for Health and Care Excellence highlighted that evidence for the effectiveness of financial incentives in a UK context was needed. ${ }^{8}$ The latest Cochrane systematic review of this topic suggests that financial incentives may help pregnant smokers to stop but that the evidence is limited. ${ }^{12}$ A recently completed mixed methods study to inform trial design about the benefits of incentives ${ }^{13}$ concluded that financial incentives may be appropriate to support smoking cessation during pregnancy, with a lower limit of effectiveness for cessation at $£ 20$ a month and an upper limit of acceptability at $£ 80$ a month.

We carried out a single centre, randomised controlled trial in Glasgow, Scotland, to assess acceptability and begin to explore efficacy and cost effectiveness of up to $£ 400$ of shopping vouchers added to routine specialist pregnancy NHS "Stop Smoking Services" to help pregnant smokers quit.

\section{Methods \\ Design}

This was a phase II therapeutic exploratory single centre, individually randomised controlled parallel group superiority trial with 1:1 allocation and single blinding of initial primary outcome assessment. The trial design also included a process evaluation involving qualitative methods and an economic analysis. These elements of the trial are reported elsewhere. ${ }^{14}$

\section{Study population}

Although described as a single centre, NHS Greater Glasgow and Clyde has the largest health board population $(1.2 \mathrm{~m})$ in the United Kingdom. It is spread over a wide geographical area including severely materially deprived post-industrial areas as well as some more affluent communities. Maternity booking and antenatal care are provided in both hospital and local healthcare settings. Delivery (13 844 infants in $2013^{15}$ ) takes place in three major maternity hospitals.

At maternity booking all Glasgow women are asked about current smoking (at least one cigarette in the preceding week), and nearly all (97\%) consent to a carbon monoxide breath test. ${ }^{16}$ Self reported smokers are routinely notified to the NHS stop smoking services. During trial recruitment (December 2011 to February 2013), in addition to routine care, advisers from the stop smoking services sought permission to pass women's contact details to the trial team. Women were eligible if they were smokers with an exhaled carbon monoxide level of at least 7 ppm, aged 16 years or more, less than 24 weeks pregnant, resident in NHS Greater Glasgow and Clyde, and able to understand and speak English for telephone consent. A carbon monoxide level of $7 \mathrm{ppm}$ indicates current smoking, although $38 \%$ of self reported smokers at maternity booking have levels below this value ${ }^{17}$ often because they have not smoked since the preceding evening or are light smokers.

\section{Study protocol and interventions}

The study followed a published protocol,${ }^{18}$ which was approved by West of Scotland research ethics committee 2 on 25 May 2011. A substantial amendment to consent, passed on 15 August 2012, allowed use of residual routine late pregnancy blood samples to be tested for cotinine (that is, samples collected as part of routine antenatal care rather than specifically for the trial). We sought samples for the last 200 participants enrolled. This provided a check on the primary outcome for this group of participants and assessed smoking status of those lost to follow-up.

\section{Enrolment}

The stop smoking services aim to contact all smokers notified to them. Throughout the enrolment period, advisers from the services discussed the trial with referred women during their first contact. The contact details of clients who gave permission were securely passed to the trial team and helpline staff through the trial database. The helpline (a call centre also commissioned to provide the NHS Smoking Helpline) contacted women, confirmed that all selection criteria had been met, enrolled participants using telephone consent, and conducted concealed random allocation. All contacts with the helpline were audio recorded. The trial database and web portal, developed by Echo Managed Services, allowed the trial team and helpline staff to enter data at separate sites.

\section{Randomisation and masking}

We randomly allocated eligible women to either receive the offer of up to $£ 400$ of shopping vouchers (if they engaged with services and subsequently quit) added to routine specialist pregnancy stop smoking services or routine care alone. The Glasgow Clinical Trials Unit embedded the randomisation in the trial database using randomised permuted blocks, with a block length of four, thus facilitating equal distribution of clients between the interventions. Allocation was concealed from staff and clients until after consent and recruitment. Helpline staff, who ascertained the primary outcome, self report of smoking status in late pregnancy, were blind to allocation status.

\section{Routine specialist pregnancy stop smoking services}

All smokers identified at maternity booking are electronically notified to stop smoking services. The service's advisers try to make contact, and smokers can opt out at this point. The advisers offer a one hour appointment to discuss smoking and cessation. This face to face contact is followed by four weekly telephone support calls and free nicotine replacement therapy from local pharmacies for 10 weeks. Contact to ascertain smoking status is attempted at four weeks, 12 weeks (if quit at four weeks) and one year after the agreed quit date. 


\section{Study groups}

The control group was offered routine specialist pregnancy support by the stop smoking services. The incentives group was offered the same routine support plus up to $£ 400$ of shopping vouchers (Love2shop) for engaging with stop smoking services or for quitting during pregnancy, or both. Intervention participants received $£ 50$ of vouchers if they attended their face to face appointment and set a quit date. As per routine practice, clients who set a quit date were telephoned by their stop smoking services adviser four weeks later. Intervention participants who reported abstinence (that is, had not smoked, even a puff, in the past two weeks) were visited at home by a research nurse to confirm smoking status (carbon monoxide breath test result $<10$ ppm). Confirmed quitters were sent a further $£ 50$ voucher. Twelve weeks after stopping smoking, women in the incentives group who were quitters at four weeks were contacted by stop smoking services (routine practice) and, if confirmed to be abstinent (carbon monoxide breath test result $<10 \mathrm{ppm}$ ), were sent a $£ 100$ voucher. At a randomly allocated time between 34 and 38 weeks' gestation all participants were contacted by the helpline staff to ascertain smoking status. A research nurse visited self reported quitters to collect a carbon monoxide level, and saliva and urine for cotinine estimation. Women in the incentives group who were confirmed as abstinent by the carbon monoxide breath test $(<10 \mathrm{ppm})$ were sent a final $£ 200$ voucher. An external fulfilment house linked to the trial database sent all vouchers directly to participants.

\section{Data collection}

We collected baseline information on carbon monoxide readings at maternity booking; self reported smoking status; postcode of residence; estimated date of delivery; maternal age, height, and weight; English speaking; the Fagerstrom questionnaire to assess nicotine addiction ${ }^{19}$; partner smoking status; parity; and ethnicity.

Data collected at subsequent visits were:

First visit to stop smoking services-date, attended or not, quit date.

Four week post quit contact-smoked even a puff in past two weeks, carbon monoxide level for self reported quitters in the incentives group.

12 week post-quit contact for intervention group quitters confirmed by carbon monoxide reading at 4 weeks — smoked even a puff in past four weeks, carbon monoxide level for self reported quitters in the incentives group.

All participants at 34-38 weeks' gestation, May 2012 to September 2013-smoked fewer than five cigarettes in the past eight weeks, current use of nicotine replacement therapy, carbon monoxide level, and saliva and urine cotinine level for all self reported quitters. The main primary outcome analysis assumed that participants who were lost to follow-up (that is, self reported smoking status or validation of urine and saliva samples not obtained) at 34-38 weeks' gestation had continued to smoke. To assess if this assumption was valid, we gathered the residual routine late pregnancy blood samples taken from the last 200 participants enrolled in the trial, collected from NHS laboratories, and assayed them for cotinine. To minimise losses to follow-up, particularly among controls, a £25 shopping voucher was sent to all participants if they provided primary outcome information and saliva or urine samples as appropriate. Six month postnatal contact for cotinine confirmed quitters in late pregnancy, December 2012 to March 2014 —still quit or smoked as few as five cigarettes in total since their quit date (at least 12 months post-quit).
All participants — date of delivery, birth weight, stillbirth, or miscarriage (to assess any potential harms).

\section{Outcomes}

Table $1 \Downarrow$ describes the registered and non-registered outcomes. Non-registered outcomes stillbirth and miscarriage were described as adverse events to be documented in the protocol (see table $1 \Downarrow$ ). ${ }^{18}$ We collected gestation used to define preterm ( $<37$ completed weeks' gestation) as a confounding variable for birth weight. Towards the end of the trial, funders (Glasgow Centre for Population Health) suggested that intervention women may be more likely to take up smoking again soon after their child was born. Longer term health economic analysis indicated that this would be important in determining cost per quality adjusted life year gained. We therefore obtained self report of current smoking status 12 months after quit date-about six months after birth-from data routinely collected by stop smoking services.

\section{Statistical analysis}

Assuming a quit rate of $4 \%$ in the control group, ${ }^{20} 600$ participants gave $90 \%$ power to detect an increase in quit rate to $11.4 \%$, or $80 \%$ power to detect an increase in quit rate to $10.2 \%$, based on a continuity corrected $\chi^{2}$ test at $5 \%$ significance. We have summarised continuous data as means (standard deviations) and categorical data as counts (percentages), with $t$ tests or Fisher's exact tests used, respectively, to compare between group comparisons. Smoking status outcomes are presented as the relative risk ( $95 \%$ confidence interval) of not smoking. We used logistic regression models to control for baseline smoking dependence (Fagerstrom questionnaire) and deprivation status. Analysis of outcomes was by intention to treat, with 5\% statistical significance. Given the definition of the primary outcome, those who did not provide outcome data were not eligible to receive incentives and were, by default, treated as being a smoker at follow-up. There was therefore no missing data in the primary analysis. Analyses were performed using SAS (v9.3).

\section{Results}

\section{Participant flow and follow-up}

Of 3052 self reported smokers identified at maternity booking between December 2011 and February 2013, 1128 (37\%) were ineligible as their carbon monoxide reading was $<7 \mathrm{ppm}$. A further 898 (29\%) were ineligible, mainly because stop smoking services were unable to make contact with them. Only 124 of 1150 eligible women contacted $(11 \%)$ refused permission for their contact details to be passed to the trial team. Details for 1026 women were therefore transferred (figure $\Downarrow$ ). Of this 1026 , after at least three attempts (at times chosen by the client) by the trial team, 379 (37\%) women could not be contacted. In addition, seven women had miscarried and contact was not attempted. Of the 640 women contacted, eight were no longer pregnant and 20 declined to participate. The trial population of 612 pregnant smokers represented $20 \%$ of all self reported smokers $(n=3052)$ who attended for maternity care during the study period, and 53\% of $1150(1026+124)$ eligible pregnant smokers who could be contacted by advisers as part of routine stop smoking services (figure).

Overall, 306 participants were allocated to the incentives group and 306 to the control group. Table $2 \Downarrow$ shows the baseline characteristics. The two groups were well balanced, except that 
control participants were slightly more nicotine dependent, with a higher Fagerstrom score. ${ }^{19}$

Three control participants withdrew from the study immediately after randomisation and did not wish any of their data to be included in the statistical analysis. At the primary outcome assessment call, two intervention participants withdrew from the study. These participants were included in the statistical analysis, leaving 306 intervention and 303 control participants in the final analysis (table $3 \Downarrow$ and figure).

\section{Primary outcome}

Significantly more smokers offered incentives had stopped smoking at primary outcome assessment: 69 (22.5\%) versus 26 $(8.6 \%)$. The relative risk of not smoking at the end of pregnancy was 2.63 (95\% confidence interval 1.73 to 4.01 ), $\mathrm{P}<0.001$ (table 3 ). These results were unaffected after controlling for nicotine dependence. The absolute risk difference was 14.0\% (95\% confidence interval $8.2 \%$ to $19.7 \%$ ). The number needed to treat was 7.2 (95\% confidence interval 5.1 to 12.2$)$.

Forty three (14\%) control and $46(15 \%)$ intervention participants were lost to follow-up at primary outcome assessment.

\section{Secondary outcomes}

There was a higher rate of self reported abstinence from smoking at four weeks after the quit date in the incentives group than control group (table $4 \Downarrow$ ). No between group differences were seen for engagement with stop smoking services, birth weight, still birth, miscarriage, or premature birth. Women who were validated as abstinent by cotinine measurement towards the end of pregnancy were followed up by telephone postnatally at least 12 months after their original quit date; self report data collected at this point continued to show a large difference ( $15 \%$ v 4\%) in abstinence rates between the incentives and control groups. With regard to postnatal relapse, of 26 cotinine validated quitters in the control group, three could not be contacted and 11 self reported as relapsed ( $42 \%$ of cotinine validated quitters). Of 69 quitters in the incentives group, four could not be contacted and 18 self reported as relapsed ( $26 \%$ of cotinine validated quitters). Health economic outcomes are reported in full elsewhere. ${ }^{14}$

\section{Residual routine blood sample analysis}

Analysis of residual routine pregnancy samples that were available from the last 200 women in the trial indicated that clients who could not be reached or fully assessed for the primary outcome were likely to be smokers (table 3 ). This validates the assumption made within the main analysis that participants who were lost to follow-up at the primary outcome collection point at 34-38 weeks' gestation were still smoking.

Firstly, for 46 participants in the incentives group, contact could not be made at 34-38 weeks' gestation after multiple attempts, initially by the helpline and then by research nurses after contact checks. Ten had residual blood samples available, taken for other purposes at 32-42 weeks' gestation, and all 10 samples indicated current smoking when tested for cotinine. Similarly, three residual samples were available from 43 control participants, and all indicated current smoking.

Secondly, 30 participants in the incentives group who self reported as quit at 34-38 weeks' gestation could never be reached to allow research nurses to collect a carbon monoxide sample to validate their self report and to allow the final $£ 200$ incentive payment to be sent. Two residual blood samples were available from this group, and both indicated current smoking. Likewise, 23 self reported quitters in the control group were never available for corroboration samples to be collected and therefore did not receive the $£ 25$ incentive payment. No residual blood samples were available from this group.

Finally, 69 participants in the incentives group had stopped smoking in late pregnancy, as defined by the Russell Standard ${ }^{21}$ primary outcome of self report at 34-38 weeks' gestation corroborated by measurement of saliva or urine cotinine. From these participants, 18 had residual blood samples collected for other reasons in late pregnancy (32-42 weeks' gestation) available for cotinine analysis. Eighty per cent (samples from 14 women) confirmed that the participant had quit smoking in late pregnancy. Similarly, from 26 control participants who quit, five residual samples were available and four $(80 \%)$ confirmed abstinence. This suggests that a small proportion of women to whom we allocated a positive primary outcome may have returned to smoking, at least occasionally, within the last 10 weeks of pregnancy, but relapse rates were similar in both groups and hence this was unlikely to have affected the principal findings.

More importantly, this additional analysis of residual samples confirmed that $80 \%$ of those who were defined as quitters had truly quit smoking by late pregnancy and were not "gaming" the primary outcome assessment.

\section{Discussion}

This trial indicates that financial incentives can motivate pregnant smokers to quit. Significantly more smokers offered incentives stopped smoking (22.5\%) than controls (8.6\%). This study is the largest so far undertaken to test financial incentives to help pregnant women stop smoking. The four studies (three in the United States, one in the United Kingdom) included in the BIBS (benefits of incentives for breastfeeding and smoking cessation in pregnancy) systematic review 2014 of financial incentives for smoking cessation in pregnancy ${ }^{13}$ included 332 participants in total. The combined effect size was a relative risk for quitting of 2.58 (95\% confidence interval 1.63 to 4.07 ). This present trial included 612 participants and backs up the findings of the previous smaller studies, with a relative risk of 2.63 (95\% confidence interval 1.73 to 4.01 ). The absolute risk difference was $14.0 \%$ (95\% confidence interval $8.2 \%$ to $19.7 \%$ ), meaning that the number needed to treat (NNT) was $7.2(95 \%$ confidence interval 5.1 to 12.2). It should be made clear that the intervention is the offer of financial incentives if cessation is achieved. Therefore the NNT is the number of pregnant smokers who need to be offered financial incentives for each extra quitter in late pregnancy, not the number who need to be given financial incentives. This improvement in smoking cessation is larger than that seen in most behavioural ${ }^{12}$ or pharmaceutical ${ }^{22}$ trials of smoking cessation during pregnancy. Previous systematic reviews ${ }^{12}$ highlight that current recommendations to help smokers quit during pregnancy ${ }^{8}$ are not very effective.

\section{Strengths of this study}

The trial included a process evaluation and an economic analysis, the results of which are reported elsewhere. ${ }^{14}$ No harms were reported, and incentives were acceptable to clients and healthcare professionals. The short term incremental cost per quitter at 34-38 weeks' gestation was £1127, and longer term cost per quality adjusted life year gained was $£ 482 .{ }^{14}$ The latter is well below the UK National Health Service threshold of $£ 20$ $000 .^{23}$

One concern was that more women randomised to the control group (that is, no incentives) might be unavailable for primary 
outcome assessment. This did not happen, as attrition in both groups was similar. Forty three $(14.2 \%)$ participants in the control group and $46(15 \cdot 1 \%)$ in the incentives group could not be contacted towards the end of pregnancy. Those lost to follow-up were assumed to be smokers. We were able to test this assumption. From the last 200 participants, routine residual late pregnancy blood samples were available from three control and 10 intervention participants lost to follow-up. All were validated as smokers by measurement of cotinine. This supports the assumption in the analysis that those lost to follow-up were smokers.

Another issue is the extent to which participants were representative of the local population. We tested this and results have been submitted for publication. Trial participants were representative of all self reported smokers at maternity booking in Glasgow.

A possible unintended consequence of financial incentives was that women were untruthful about their smoking status when asked during the trial, especially at the time of the primary outcome assessment. Testing for this type of "gaming" was not the main focus of the trial but was examined to a limited extent. We found some evidence of women being untruthful about their smoking status over the telephone. Eighteen participants who self reported as quit to the helpline subsequently reported as current smokers when arranging a confirmation visit with the research nurse. Fifty three others were never available to the research nurses to collect confirmatory samples. Of these, two had routine residual late pregnancy blood samples available, both from intervention participants, which confirmed current smoking. Secondly, a small number of self reported quitters were validated as non-smokers by carbon monoxide breath testing but may have only temporarily abstained, as $18(16 \%)$ were confirmed as current smokers by testing of saliva or urine collected by the research nurses at the primary outcome home visit: six (19\%) controls and $12(15 \%)$ intervention participants. Finally, $4 / 18$ intervention and $1 / 5$ control group quitters in late pregnancy confirmed by cotinine measurement who also had a residual routine blood sample taken (table 3), were confirmed as being positive for cotinine. These women may not have truly quit smoking in late pregnancy and this issue merits further research.

Incentives for health behaviour change remain controversial. ${ }^{24}$ Public perceptions of "paying" individuals to change behaviour can be negative. However, qualitative elements found that incentives were acceptable to women and healthcare professionals. ${ }^{14}$ In addition, the level of incentive was tested in a public opinion survey conducted as part of a related study and was deemed acceptable. ${ }^{13}$

\section{Weaknesses of this study}

This was a phase II study, a therapeutic exploratory trial ${ }^{25}$ conducted in one centre to explore the efficacy of a new intervention. Although this was a large phase II trial, a future definitive trial is clearly needed in more than one centre to confirm efficacy, cost effectiveness, and generalisability. A future study needs to consider geographical location and type of smoking cessation service-for example, specialist pregnancy service versus generic cessation service and specialist practitioners versus pharmacy services. ${ }^{26}{ }^{27}$ In addition, the economic analysis included some uncertainty in the results driven by postnatal relapse. Future studies should more closely examine outcomes in the postnatal period.

The registered secondary outcome, cessation at four weeks (table 4), available from routine telephone follow-up at four weeks with the NHS stop smoking services, had high losses to follow-up-105 (34\%) in the incentives group and 171 (56\%) in the control group. This number included $58(19 \%)$ intervention and 67 (22\%) control participants who did not engage at all with the stop smoking services. Using routinely collected service data often leads to greater losses to follow-up. However, overall quit rates at four weeks post-quit date reflected the same pattern of change in cessation between incentives and control groups (doubling of quit rate) that was seen in the primary outcome at 34-38 weeks' gestation.

\section{Ethical issues related to financial incentives}

Smoking during pregnancy remains a major health problem, resulting in the deaths of an estimated 5000 fetuses and babies each year in the United Kingdom ${ }^{1-6}$ and is responsible for tens of millions of pounds in extra healthcare spending. ${ }^{8}$ The research question for this study was originally put forward by the National Institute for Health and Care Excellence. ${ }^{8}$ Financial incentives are a complex topic, with several ethical considerations. However, in addition to effectiveness, an important outcome is cost effectiveness, particularly the longer term cost per quality adjusted life year gained, taking account of any "gaming." NICE will then decide, using the standard cut-off of $£ 20000$ per quality adjusted life year gained, if, as with a new drug or device, this intervention should be recommended for use by the NHS in the United Kingdom. In this study, we assessed a new public health intervention (financial incentives) using clinical trial methods ${ }^{25}$ often reserved for new drug products or devices. Most drugs are developed and used once a disease or condition has manifested and the patient has sought advice from a doctor. Only a few interventions, such as vaccines, are able to prevent disease. Financial incentives to stop smoking could reach smokers who become pregnant before cigarettes have affected their health. The reduced mortality and morbidity among offspring will be multiplied if children subsequently do not take up smoking because their mother stopped before they were born and thus lessened the risk that they would grow up in a smoking household. In the developed world there is now a clear socioeconomic gradient in smoking, with tobacco use concentrated among the poorest in society. Receipt of financial incentives can contribute to needed household income in advance of the arrival of a baby in low income households. In addition, stopping smoking reduces inequalities in health outcomes. If financial incentives are effective and cost effective they may well have the future potential to sit with vaccines as an important preventive healthcare intervention strategy.

\section{Conclusions}

Smoking in pregnancy remains a leading preventable cause of maternal and neonatal ill health and death in the United Kingdom and in most other developed countries. Existing interventions are not highly effective. This study provides substantial evidence of a promising and potentially cost effective new intervention to add to present health service support. The findings can serve as the basis for future research to include other UK centres and other healthcare systems.

Other members of the Cessation in Pregnancy Incentives Trial (CPIT) Team are: Andrew Briggs, chair in health economics, Health Economics and Health Technology Assessment Unit, University of Glasgow; Alan Cameron, honorary professor, obstetrics and gynaecology, School of Medicine, University of Glasgow; Liz Grant, lead public health pharmacist (health improvement); Margaret McFadden, research nurse; Anne 


\section{What is already known on this topic}

Financial incentives have been used in several small trials to help pregnant smokers to quit in the United States, and the National Institute for Health and Care Excellence has called for research in the United Kingdom to examine cost effectiveness

A recent mixed methods study to inform trial design included a systematic review, which estimated the relative risk of cessation to be 2.58 (95\% confidence interval 1.63 to 4.07 ) with financial incentives

A discrete choice experiment using a MORI poll of the general UK population suggested effective and acceptable levels of incentive payment lay between $£ 20$ and $£ 80$ per month

\section{What this study adds}

In this single centre therapeutic exploratory UK trial, $£ 400$ of voucher incentives paid in instalments over six months helped pregnant smokers to quit, with no adverse effects and little evidence of "gaming"

Methodology has been developed to run a definitive multicentre trial to examine generalisability and cost effectiveness in the United Kingdom

Benson, project assistant, NHS Greater Glasgow and Clyde; Sue Stevenson, research nurse, University of Glasgow; Carol Anne Greenan, research administrator, University of Stirling; Caroline Haig, trainee biostatistician; John McHugh, clinical database manager, Robertson Centre for Biostatistics and Glasgow Clinical Trials Unit; and Janet Ferguson, trial manager (when the study was set up), University of Stirling.

Contributors: DT, LB, LdeC, CT, AM, KB, SM, and JM conceived and designed the work. LS managed the trial and, along with $\mathrm{BF}, \mathrm{SM}$, and JM collected the data. KB, DP, SM, JM, and AM analysed the data. AR, TC, DT, LB, and AM interpreted the data. All authors drafted the work or revised it critically for important intellectual content, gave final approval of the version to be published, and agree to be accountable for all aspects of the work in ensuring that questions related to the accuracy or integrity of any part of the work are appropriately investigated and resolved. DT and LB are the guarantors.

Funding: The primary funder was the Chief Scientist Office, Scottish Government. Two additional main funders were the Glasgow Centre for Population Health and the Education and Research Endowment Fund of the Director of Public Health Greater Glasgow and Clyde health board. Additional funders were the Yorkhill Children's Charity and the Royal Samaritan Endowment Fund. The funders had no role in writing the manuscript or the decision to submit it for publication. No payment from a pharmaceutical or any other company has been paid to write this article. The corresponding author had full access to all the study data and had the final responsibility for the decision to submit for publication. TC acknowledges the support of East Midlands Collaboration for Leadership in Applied Health Research and Care for his work in general, but no payment was received by him specifically for this study. Competing interests: All authors have completed the ICMJE uniform disclosure form at www.icmje.org/coi_disclosure.pdf and declare: no support from any organisation for the submitted work; no financial relationships with any organisations that might have an interest in the submitted work in the previous three years; no other relationships or activities that could appear to have influenced the submitted work.

Ethical approval: This study was approved by West of Scotland research ethics committee 2 .

Data sharing: Glasgow University in conjunction with the UK Centre for Tobacco and Alcohol Studies (Stirling University) and ECHO Managed Services, owners of the database are willing to examine all requests for the dataset on an individual basis after a period of three years from the date of this publication. Access to data will be formalised on a contractual basis with the UK Centre for Tobacco and Alcohol Studies in conjunction with Glasgow and Stirling Universities and ECHO Managed Services. The research protocol is available on request from the first author (david.tappin@glasgow.ac.uk). The protocol has been published: Tappin DM, Bauld L, Tannahill C, de Caestecker L, Radley A, McConnachie A, et al. The Cessation in Pregnancy Incentives Trial (CPIT): study protocol for a randomised controlled trial. Trials 2012;13:113.
Transparency: The lead author (DT) affirms that this manuscript is an honest, accurate, and transparent account of the study being reported; that no important aspects of the study have been omitted; and that any discrepancies from the study as planned (and, if relevant, registered) have been explained.

1 Jurkovic D, Overton C, Bender-Atik R. Diagnosis and management of first trimester miscarriage. BMJ 2013;346:\{3676.

2 Health and Social Care Information Centre, Infant feeding survey 2010. HSCIC, 2012. www.hscic.gov.uk/pubs/ifs2005.

3 US Department of Health and Human Services. The health consequences of smoking - 50 years of progress: a report of the surgeon general. US Department of Health and Human Services, Centers for Disease Control and Prevention, National Center for Chronic Disease Prevention and Health Promotion, Office on Smoking and Health, Chapter 9. 2014. www. surgeongeneral.gov/library/reports/50-years-of-progress/sgr50-chap-9.pdf.

4 Flenady V, Koopmans L, Middleton P, Frøen JF, Smith GC, Gibbons K, et al. Major risk factors for stillbirth in high-income countries: a systematic review and meta-analysis. Lancet 2011;377:1331-40.

5 Office for National Statistics. Infant and perinatal mortality in England and Wales by socia and biological factors. Stat Bull 2011, Key findings. www.ons.gov.uk/ons/dcp171778 300596.pdf.

6 Dietz PM, England LJ, Shapiro-Mendoza CK, Tong VT, Farr SL, Callaghan WM. Infant morbidity and mortality attributable to prenatal smoking in the U.S. Am J Prev Med 2010;39:45-52.

7 Gray R, Bonellie SR, Chalmers J, Greer I, Jarvis S, Kurinczuk J, et al. Contribution of smoking during pregnancy to inequalities in stillbirth and infant death in Scotland 1994-2003: retrospective population based study using hospital maternity records. BMJ 2009;339:b3754.

8 National Institute for Health and Care Excellence. Quitting smoking in pregnancy and following childbirth. 2010. http://guidance.nice.org.uk/PH26.

9 NHS Quality Improvement Scotland. Scottish perinatal and infant mortality and morbidity report 2004. Scottish Programme for Clinical Effectiveness in Reproductive Health, 2005:35. www.isdscotland.org/isd/files/mat spimmr2004_2.pdf.

10 Information Services Division, NHS National Services Scotland. Births and babies: smoking and pregnancy, 2009. www.isdscotland.org/isd/2911.html.

11 Tappin DM, MacAskill S, Bauld L, Eadie D, Shipton D, Galbraith L. Smoking prevalence and smoking cessation services for pregnant women in Scotland. Subst Abuse Treat Prev Policy 2010;5:1.

12 Chamberlain C, O'Mara-Eves A, Oliver S, Caird JR, Perlen SM, Eades SJ, et al. Psychosocial interventions for supporting women to stop smoking in pregnancy. Cochrane Database Syst Rev 2013;10:CD001055.

13 Hoddinott P, Hislop J, Morgan H, Stewart F, Farrar S, Rothnie K, et al. Incentive interventions for smoking cessation in pregnancy: a mixed methods evidence synthesis. Lancet 2012;380:S48.

14 Tappin D, Bauld L, Purves D, Boyd K, Sinclair L, MacAskill S, et al. Financial incentives for smoking cessation during pregnancy: a randomised controlled trial. Lancet abstract [forthcoming]. www.thelancet.com/pdfs/journals/lancet/PIIS0140-6736\%2814\%2962130-

15 Information Services Division Scotland. 2014. https://isdscotland.scot.nhs.uk/HealthTopics/Maternity-and-Births/Publications/2014-08-26/2014-08-26-Births-Report.pdf? 99891299010.

16 Director of Public Health, Greater Glasgow and Clyde NHS Board. Update on progress with recommendations from the Director of Public Health report: keeping health in mind. Board paper 12/52. 2014. http://library.nhsggc.org.uk/mediaAssets/Board\%20Papers/1252.pdf.

17 Usmani ZC, Craig P, Shipton D, Tappin D. Comparison of CO breath testing and women's self-reporting of smoking behaviour for identifying smoking during pregnancy. Subst Abuse Treat Prev Policy 2008:3:4

18 Tappin DM, Bauld L, Tannahill C, de Caestecker L, Radley A, McConnachie A, et al. The Cessation in Pregnancy Incentives Trial (CPIT): study protocol for a randomised controlled trial. Trials 2012;13:113.

19 Heatherton TF, Kozlowski LT, Frecker RC, Fagerstrom KO. The Fagerstrom test for nicotine dependence: a revision of the Fagerstrom Tolerance Questionnaire. Br J Addict 1991;86:1119-27.

20 Tappin DM, Lumsden MA, Gilmour WH, Crawford F, McIntyre D, Stone DH, et al. Randomised controlled trial of home based motivational interviewing by midwives to help pregnant smokers quit or cut down. BMJ 2005;331:373-7.

21 West R, Hajek P, Stead L, Stapleton J. Outcome criteria in smoking cessation trials: proposal for a common standard. Addiction 2005;100:299-303.

22 Coleman T, Chamberlain C, Davey MA, Cooper SE, Leonardi-Bee J. Pharmacologica interventions for promoting smoking cessation during pregnancy. Cochrane Database Syst Rev 2012;9:CD010078. 
23 National Institute for Health and Care Excellence. Guide to the methods of technology appraisal: 2013. http://publications.nice.org.uk/guide-to-the-methods-of-technologyappraisal-2013-pmg9.

24 Jochelson K. Paying the patient: improved health using financial incentives King's Fund, 2007. www.kingsfund.org.uk/sites/files/kf/field/field_document/paying-the-patient-kickingbad-habits-supporting-paper-karen-jochelson.pdf.

25 International Conference on Harmonisation of Technical Requirements for Registration of Pharmaceuticals for Human Use. General considerations for clinical trials, 1997. Guideline E8, section 3.1.3.2. www.ich.org/products/guidelines/efficacy/efficacy-single/ article/general-considerations-for-clinical-trials.html.

26 McGowan A, Hamilton S, Barnett D, Nsofor M, Proudfoot J, Tappin DM. 'Breathe': the stop smoking service for pregnant women in Glasgow. Midwifery 2010;26:e1-13.
27 Radley A, Ballard P, Eadie D, MacAskill S, Donnelly L, Tappin D. Give It Up For Baby: outcomes and factors influencing uptake of a pilot smoking cessation incentive scheme for pregnant women. BMC Public Health 2013;13:343.

\section{Accepted: 9 December 2014}

Cite this as: BMJ 2015;350:h134

This is an Open Access article distributed in accordance with the Creative Commons Attribution Non Commercial (CC BY-NC 4.0) license, which permits others to distribute, remix, adapt, build upon this work non-commercially, and license their derivative works on different terms, provided the original work is properly cited and the use is non-commercial. See: http://creativecommons.org/licenses/by-nc/4.0/. 


\section{Tables}

\section{Table 1| Details of registered and non-registered outcomes}

\begin{tabular}{|c|c|c|c|c|}
\hline Outcomes & Timing & Outcome evaluation method & Registered & Non-registered \\
\hline \multicolumn{5}{|l|}{ Primary outcome: } \\
\hline Quit in late pregnancy & 34-38 weeks' gestation & $\begin{array}{l}\text { Cotinine verified (saliva }<14.2 \mathrm{ng} / \mathrm{mL} \text { or urine }<44.7 \mathrm{ng} / \mathrm{mL} \text { ) self } \\
\text { report of "Have you smoked in the last eight weeks?" If yes "Have } \\
\text { you smoked more than five cigarettes in that time?" Russell } \\
\text { Standard outcome }\end{array}$ & ( & - \\
\hline \multicolumn{5}{|l|}{ Secondary outcomes: } \\
\hline Cost effectiveness* & & Framework developed to assess costs and benefits & - & - \\
\hline Engagement & 0-4 weeks after enrolment & $\begin{array}{l}\text { Attended face to face appointment with NHS stop smoking } \\
\text { services and set a quit date }\end{array}$ & ( & - \\
\hline Quit at 4 weeks & 4-9 weeks after enrolment & $\begin{array}{l}\text { Self report through telephone contact from NHS stop smoking } \\
\text { services of "Have you smoked in the last } 2 \text { weeks, even a puff?" }\end{array}$ & प & - \\
\hline Quit at 6 months after birth & $\begin{array}{l}\text { Approximately } 12 \text { months } \\
\text { after quit date }\end{array}$ & $\begin{array}{l}\text { Self report through telephone contact from NHS stop smoking } \\
\text { services of "Are you still quit?" or "Have you smoked as few as } \\
\text { five cigarettes in total since you quit?" }\end{array}$ & - & Q \\
\hline Birth weight & At birth & Patient records & 口 & - \\
\hline Stillbirth & At birth & Patient records & - & प \\
\hline Miscarriage & At event & Patient records & - & $\square$ \\
\hline $\begin{array}{l}\text { Premature birth ( }<37 \text { weeks' } \\
\text { gestation) }\end{array}$ & At birth & Patient records & - & Q \\
\hline
\end{tabular}

NHS=National Health Service.

${ }^{*}$ Reported separately. 


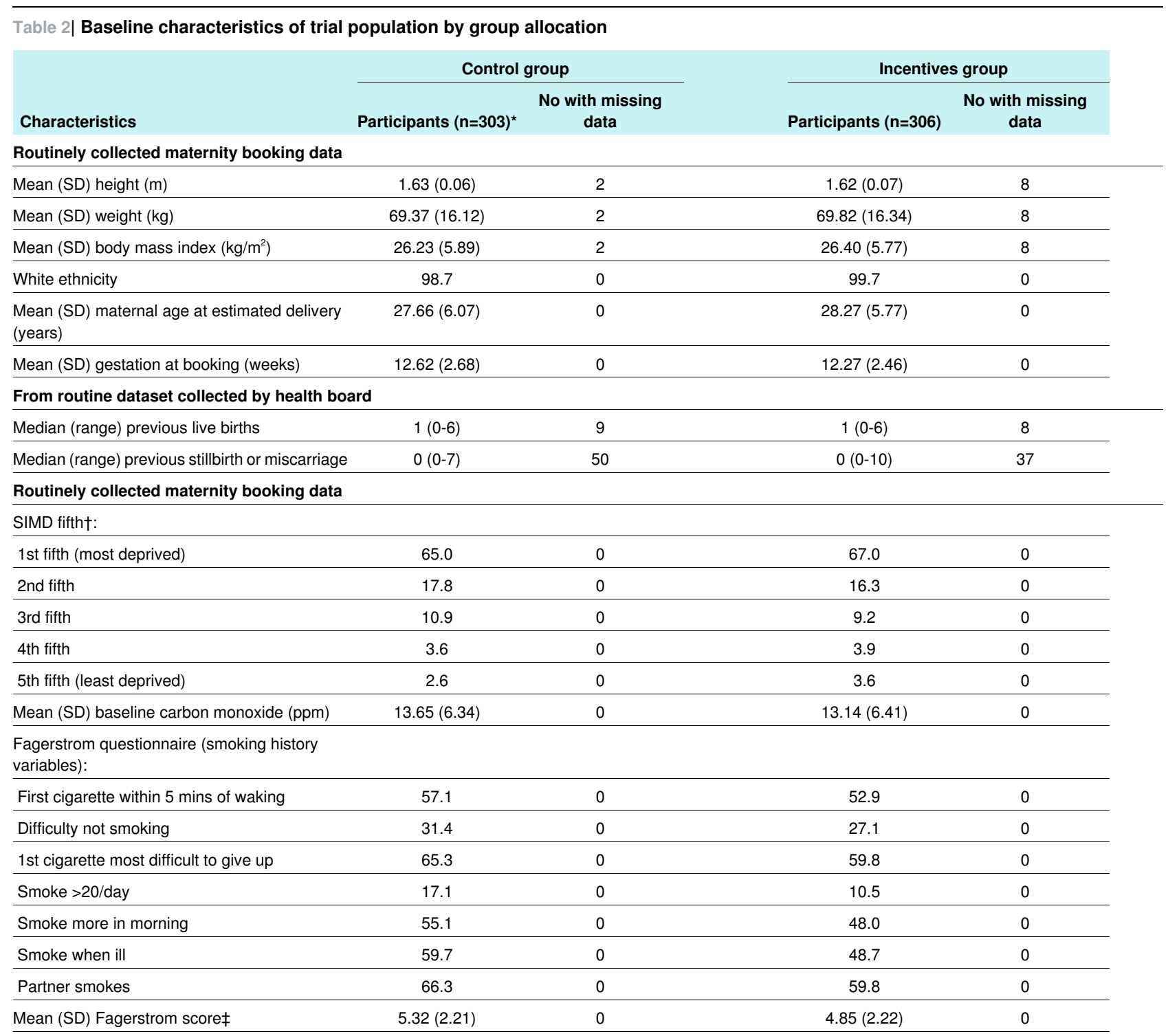

SIMD=Scottish index of multiple deprivation.

${ }^{*}$ Consent withdrawn post-randomisation for three women.

†Derived from postcode of residence.

¥Uses first six smoking history variables from Fagerstrom questionnaire. Score of $\geq 5$ indicates significant nicotine dependence. 
Table 3| Primary outcome: cotinine verified cessation at 34-38 weeks' gestation towards end of pregnancy. Values are numbers (percentages) unless stated otherwise

\begin{tabular}{|c|c|c|c|c|c|}
\hline \multirow[b]{2}{*}{$\begin{array}{l}\text { Data item, data } \\
\text { collection/analysis }\end{array}$} & \multicolumn{2}{|c|}{ Incentives group } & \multicolumn{2}{|c|}{ Control group } & \multirow{2}{*}{$\begin{array}{c}\text { Relative risk of not } \\
\text { smoking near end } \\
\text { of pregnancy (95\% } \\
\text { Cl) }\end{array}$} \\
\hline & $\begin{array}{l}\text { Participants } \\
(\mathrm{n}=306)^{\star}\end{array}$ & $\begin{array}{l}\text { Residual blood } \\
\text { cotinine estimation } \dagger\end{array}$ & $\begin{array}{l}\text { Participants } \\
\qquad(\mathrm{n}=303) \ddagger\end{array}$ & $\begin{array}{c}\text { Residual blood } \\
\text { cotinine estimation } \dagger\end{array}$ & \\
\hline \multicolumn{6}{|l|}{ Self reported smoking status } \\
\hline Smoker: & $139(45.4)$ & - & $195(64.3)$ & - & - \\
\hline Lost to follow-up & $46(15.1)$ & $\begin{array}{l}10 \text { collected (all } \\
\text { smokers) }\end{array}$ & $43(14.2)$ & $\begin{array}{l}3 \text { collected (all } \\
\text { smokers) }\end{array}$ & - \\
\hline \multicolumn{6}{|l|}{ Non-smoker: } \\
\hline When smoking status recorded & $119(46.1)$ & - & $65(25.0)$ & - & $1.84(1.44$ to 2.37$)$ \\
\hline $\begin{array}{l}\text { When loss to follow-up presumed } \\
\text { not smoking }\end{array}$ & $167(54.6)$ & - & $108(35.6)$ & - & $1.53(1.28$ to 1.84$)$ \\
\hline $\begin{array}{l}\text { When loss to follow-up presumed } \\
\text { smoking }\end{array}$ & $119(38.9)$ & - & $65(21.5)$ & - & 1.81 (1.41 to 2.35$)$ \\
\hline \multicolumn{6}{|l|}{ Saliva or urine validated smoking } \\
\hline \multicolumn{6}{|l|}{ No cotinine outcome: } \\
\hline $\begin{array}{l}\text { Changed self report to smoker } \\
\text { when phoned to arrange visit }\end{array}$ & 8 & - & 10 & - & - \\
\hline $\begin{array}{l}\text { Never contactable for carbon } \\
\text { monoxide and cotinine samples }\end{array}$ & 30 & 2 collected(2 smokers) & 23 & 0 collected & - \\
\hline $\begin{array}{l}\text { Carbon monoxide negative, } \\
\text { cotinine positive }\end{array}$ & 12 & - & 6 & - & - \\
\hline \multicolumn{6}{|l|}{ Primary outcome } \\
\hline $\begin{array}{l}\text { Self reported non-smoker and } \\
\text { saliva or urine cotinine negative }\end{array}$ & $69(22.5)$ & $14 / 18$ non-smokers & $26(8.6)$ & $4 / 5$ non-smokers & 2.63 (1.73 to 4.01$)$ \\
\hline
\end{tabular}

${ }^{*}$ Consent withdrawn post-randomisation (after 12 week stage) for two intervention women (consent given to analyse any data collected).

¥Consent withdrawn post-randomisation for three control women (consent withdrawn to utilise any data collected).

†Residual late pregnancy (between 32 and 42 weeks' gestation) blood samples collected from last 200 participants and cotinine tested. 
Table 4| Secondary outcomes and data collected. Values are numbers (percentages) unless stated otherwise

\begin{tabular}{|c|c|c|c|c|}
\hline Variables & Incentives group $(n=306)$ & Control group $(n=303)$ & Relative risk (95\% Cl) & $P$ value \\
\hline Engagement: & $248(81)$ & $236(78)$ & 1.04 (0.96 to 1.13$)$ & 0.37 \\
\hline Missing data & $0 / 306(0)$ & 0/303 (0) & & \\
\hline Quit at 4 weeks: & $133(43)$ & $64(21)$ & 2.06 (1.60 to 2.66$)$ & $<0.001$ \\
\hline Missing data (no contact) & $105(34)$ & $171(56)$ & & \\
\hline Quit at 6 months after birth: & $47(15)$ & $12(4)$ & 3.88 (2.41 to 6.23$)$ & $<0.001$ \\
\hline Missing data & $4 / 69(6)$ & $3 / 26(12)$ & & \\
\hline Mean (SD) birth weight $(\mathrm{g})$ : & $3140(600)$ & $3102(590)$ & $20(-80$ to 120$) \dagger$ & 0.67 \\
\hline Missing data* ${ }^{*}$ & 10/305 (3) & $12 / 299(4)$ & & \\
\hline Stillbirth or miscarriage: & $2(0.7)$ & $5(1.7)$ & 0.39 (0.04 to 1.99$)$ & 0.29 \\
\hline Missing data* & $5 / 305(2)$ & 3/299 (1) & & \\
\hline Premature birth: & $40(13.4)$ & $26(8.9)$ & 1.52 (0.95 to 2.39 ) & 0.09 \\
\hline Missing data* & $7 / 305(2)$ & 8/299 (3) & & \\
\hline
\end{tabular}

*Including five women (four control, one incentive) excluded from birth analyses as multiple births.

†Difference $(95 \% \mathrm{Cl})$ 


\section{Figure}

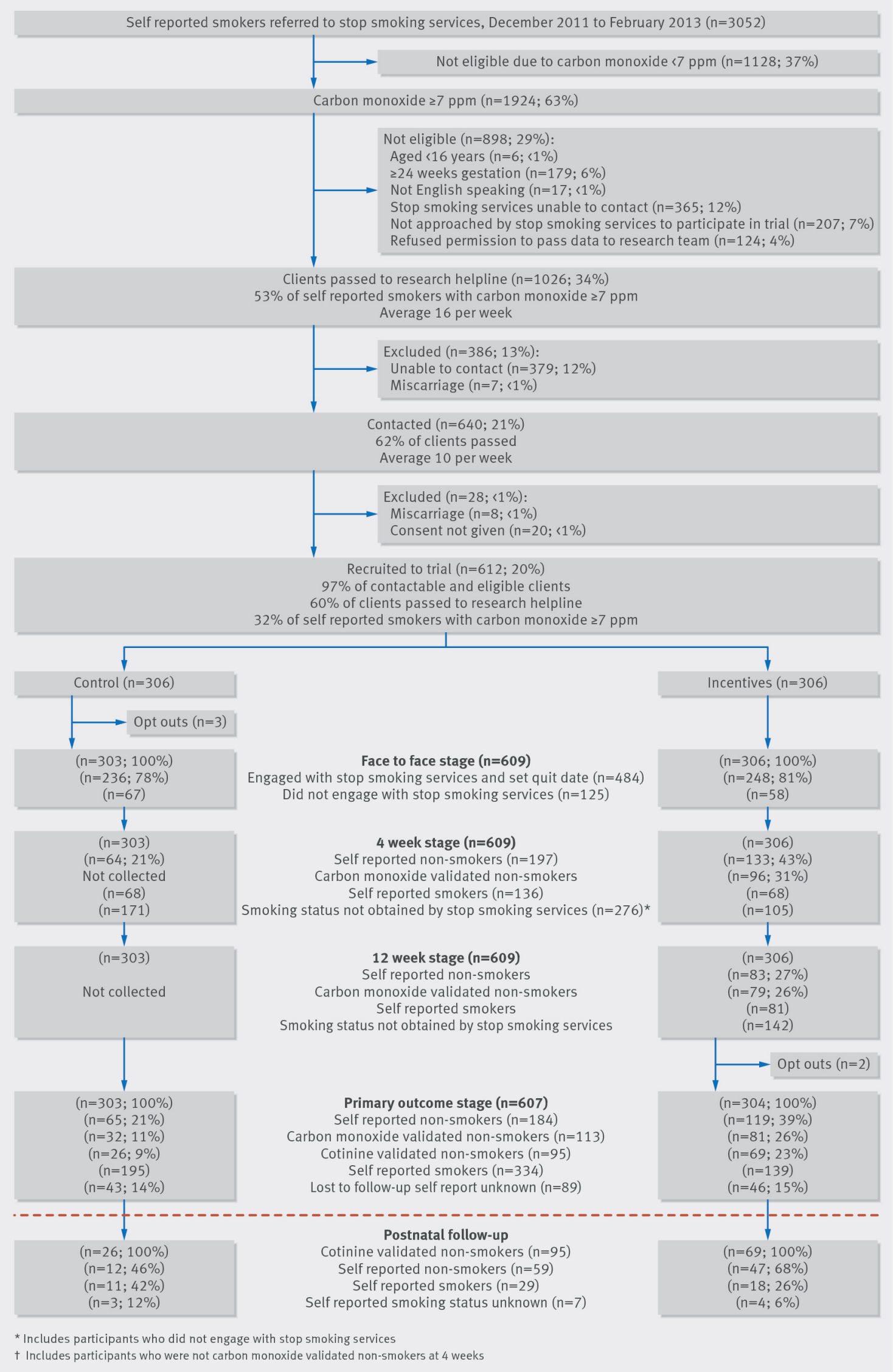

Flow of participants through study 